Gut and Liver, Vol. 10, No. 2, March 2016, pp. 262-274

\title{
Efficacy and Safety of Adalimumab in Moderately to Severely Active Cases of Ulcerative Colitis: A Meta-Analysis of Published Placebo-Controlled Trials
}

\author{
Zong Mei Zhang, Wei Li, and Xue Liang Jiang \\ Department of Gastroenterology, Chinese PLA General Hospital of Jinan Military Command, Jinan, China
}

See editorial on page 162.

Background/Aims: To evaluate the efficacy and safety of adalimumab (ADA) in moderately to severely active ulcerative colitis (UC) patients who are unresponsive to traditional therapy. Methods: Electronic databases, including the PubMed, Embase, and Cochrane databases, were searched to April 20, 2014. UC-related randomized controlled trials (RCTs) that compared ADA with placebo were eligible. Review Manager 5.1 was used for data analysis. Results: This meta-analysis included three RCTs. ADA was considerably more effective compared with a placebo, and it increased the ratio of patients with clinical remission, clinical responses, mucosal healing and inflammatory bowel disease questionnaire responses in the induction and maintenance phases $(p<0.05)$, as well as patients with steroid-free remission $(p<0.05)$ during the maintenance phase. Clinical remission was achieved in a greater number of UC cases in the ADA 160/80/40 mg groups (0/2/4 week, every other week) compared with the placebo group at week $8(p=0.006)$ and week $52(p=0.0002)$, whereas the week 8 clinical remission rate was equivalent between the ADA 80/40 mg groups and the placebo group. Among the patients who received immunomodulators (IMM) at baseline, ADA was superior to the placebo in terms of inducing clinical remission $(p=0.01)$. Between-group differences were not observed in terms of serious adverse events $(p=0.61)$. Conclusions: ADA, particularly at doses of $160 / 80 / 40 \mathrm{mg}$ (0/2/4 week, every other week), is effective and safe in patients with moderate-to-severe UC who are unresponsive to traditional treatment. Concomitant IMM therapy may improve the short-term therapeutic efficacy of ADA. (Gut Liver 2016;10:262-274)
Key Words: Adalimumab; Efficacy and safety; Meta-analysis; Randomized controlled trials; Colitis, ulcerative

\section{INTRODUCTION}

Ulcerative colitis (UC) is an idiopathic, chronic inflammatory bowel disease (IBD) and characterized by an unpredictable course of relapse and remission. ${ }^{1}$ Most patients can achieve and maintain clinical remission by 5 -aminosalicylic acid, steroids and/or oral immunomodulators (IMM) such as s azathioprine and 6-mercaptopurine conventionally. But there are still a portion of patients showed non-response to traditional treatment, and some even require surgery, which has a remarkable negative influence on patient life quality., Thus, the emergence of biological agents opens up new alternatives to the treatment of moderate-to-severe UC.

Infliximab (IFX) is the first biological agent approved to treat UC with most widely used. Subsequently, many outcomes of clinical trials ${ }^{4-9}$ showed that adalimumab (ADA) is a fully humanized IgG1 monoclonal antibody targeting to tumor necrosis factor $\alpha$ (TNF- $\alpha$ ), which can induce UC to achieve the clinical remission and maintain clinical response. In September of 2012, ADA was approved to treat moderately to severely active UC in adults who are inadequate response or intolerance to IMM by the U.S. Food and Drug Administration (FDA). ${ }^{10}$ Due to a relatively small number of clinical studies on treatments of ADA for UC, it is essential to carry out a meta-analysis on ADA as treatment for UC who are intolerant or stubborn to traditional medicine.

Correspondence to: Xue Liang Jiang

Department of Gastroenterology, Chinese PLA General Hospital of Jinan Military Command, 25 Shifanlu, Jinan 250031, China

Tel: +86-15954103609, Fax: +86-0531-88381896, E-mail: jiangxueliang678@sina.com

Received on January 25, 2015. Revised on July 28, 2015. Accepted on August 12, 2015. Published online January 19, 2016

pISSN 1976-2283 eISSN 2005-1212 http://dx.doi.org/10.5009/gnl15042

() This is an Open Access article distributed under the terms of the Creative Commons Attribution Non-Commercial License (http://creativecommons.org/licenses/by-nc/4.0) which permits unrestricted non-commercial use, distribution, and reproduction in any medium, provided the original work is properly cited. 


\section{MATERIALS AND METHODS}

\section{Search strategy}

Electronic databases, such as PubMed, Embase, Cochrane Library, were searched for researches published until April 20, 2014 containing the terms "adalimumab[Title/Abstract]" and "ulcerative colitis[Title/Abstract]" and "randomized controlled trial[MeSH]" or "random*[Title/Abstract]". For extra relevant publications, the reference lists were reviewed by hand, if a study was identified as having fulfilled the inclusive criteria previously.

\section{Study selection}

Two investigators determined if these published studies met the following inclusive criteria after screening the titles and abstracts of them independently: (1) the purpose of studies which provide the case inclusion and exclusion criteria was to access the efficacy and safety of ADA for moderate-to-severe active UC; (2) make sure that the studies are prospective randomized controlled trials (RCTs) which compare ADA therapy with the administration of a placebo; (3) patients aged $\geq 15$ years old included had to be diagnosed with UC who are stubborn to traditional treatment of steroids and/or IMM, or stubborn to intravenous corticosteroids; (4) object of studies must have been diagnosed as moderately to severely active UC, who had a Mayo score of 6 to 12, with an endoscopy subscore of 2 to $3,{ }^{11}$ and; (5) evaluation of therapeutic efficacy included one or more parameters such as short- or long-term remission, response, mucosal healing, steroid-free remission, health-related quality of life which was measured by the inflammatory bowel disease questionnaire (IBDQ), ${ }^{12,13}$ and safety of ADA.

The studies inaccessible to full research data, review, case report, letter, and editorial were excluded. Articles about children and pregnant woman were also excluded. All studies involved were published in English.

\section{Outcome assessment}

Unless the definitions of efficacy variable were given in original study, clinical remission was given a definition of a full Mayo score $\leq 2$ with no individual subscore exceeding 1 points, response was given a definition of a decrease in Mayo score $\geq 3$ points and $\geq 30 \%$ from baseline plus a decrease in the rectal bleeding subscore $\geq 1$ or an absolute rectal bleeding subscore of 0 or 1 , the endoscope subscore had to be 0 or 1 means achieving mucosal healing, IBDQ response was defined as an increase from baseline of at least 16 points, ${ }^{13,14}$ serious side effects were given definitions by each primary study. The primary efficacy end point is week 8 clinical remission rate in each group. The week 52 clinical remission rate, the rate of clinical response, mucosal healing and IBDQ respond at week 8 and 52, and the steroid-free remission rate at week 52 were valued as the secondary efficacy end points.

\section{Assessment of risk of bias}

Two investigators performed the data collection and assessment independently, wherein they resolved any disagreements by discussion. The Cochrane Handbook for Systematic Reviews of Interventions described how to evaluate the risk of bias. ${ }^{14}$ And we applied the Jadad scale ${ }^{15}$ to measure the quality of included studies, which evaluates presentations of randomization, blinding, and dropouts (withdrawals) in trials. Scale on the included studies' qualities ranks from 0 to 5 points and a low quality study scores 2 or less and a high quality literature score at least 3.

\section{Statistical analysis}

We performed the meta-analyses by using relative risk (RR) for dichotomous outcomes. The 95\% confidence intervals (CIs) were used to present pooled estimates. Subgroup analyses were chosen based upon the induction dose $(160 / 80 \mathrm{mg}$ or $80 / 40$ mg at weeks 0/2) and baseline UC medication (baseline steroids

Table 1. Baseline Characteristics of the Included Studies

\begin{tabular}{lcccccc}
\hline \multicolumn{1}{c}{ Author (year) } & $\begin{array}{c}\text { ITT patient, } \\
\mathrm{n}\end{array}$ & $\begin{array}{c}\text { Mean age, } \\
\mathrm{yr}\end{array}$ & $\begin{array}{c}\text { Male sex, } \\
\%\end{array}$ & $\begin{array}{c}\text { Intervention/ } \\
\text { control, } \mathrm{n}\end{array}$ & Cotherapy permitted & $\begin{array}{c}\text { Type of study } \\
\text { (Jadad score) }\end{array}$ \\
\hline Reinisch et al. (2011) & 390 & 37.8 & 61.9 & $260 / 130$ & CS and/or AZA or 6-MP; CS tapered & Double-blind, RCT (5) \\
Sandborn et al. (2012) & 494 & 40.4 & 57.3 & $248 / 246$ & CS and/or AZA or 6-MP; CS tapered & Double-blind, RCT (4) \\
Suzuki et al. (2014) & 273 & 42.7 & 62.7 & $177 / 96$ & CS and/or AZA or 6-MP; CS tapered & Double-blind, RCT (4) \\
\hline
\end{tabular}

ITT, intent-to-treat patients; CS, corticosteroids; AZA, azathioprine; MP, mercaptopurine; RCT, randomized controlled trial.

Table 2. Trial Design of the Included Studies

\begin{tabular}{lllcr}
\hline \multicolumn{1}{c}{ Author (year) } & \multicolumn{1}{c}{ Participant (ulcerative colitis) } & Intervention & Control group & Follow-up, wk \\
\hline Reinisch et al. (2011) & No respond to conventional therapy & Adalimumab & Placebo & 8 \\
Sandborn et al. (2012) & No respond to conventional therapy & Adalimumab & Placebo & 52 \\
Suzuki et al. (2014) & No respond to conventional therapy & Adalimumab & Placebo & 52 \\
\hline
\end{tabular}


or IMM) within the study. We calculated $\mathrm{I}^{2}$ to quantify statistical heterogeneity across trials in which $\mathrm{p}<0.10$ was determined significant. If heterogeneity was significant, that is, $\mathrm{p}<0.10$ or $I^{2}>50 \%$, we applied a random effects model to assess the total estimate. If not, a fixed effect model was used. Since studies included were not enough to make a meaningful analysis in each comparison, we did not conduct funnel plots to make investigation on publication bias. We followed the intention-to-treat principle to analyze the results.

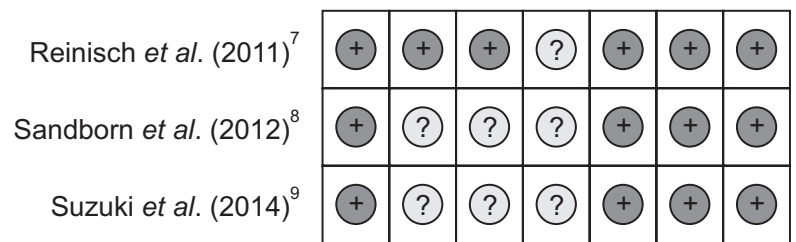

Fig. 1. Risk of bias summary.

\section{RESULTS}

\section{Characteristics of the included studies}

Fifty-nine citations were identified using the search strategy described previously, of which, 49 were eliminated after examining the title and abstract. Ten articles involving the efficacy of ADA therapy in UC were then further evaluated. Seven of these 10 articles were excluded: three trials was excluded as the study was not placebo-controlled, ${ }^{5,16,17}$ because it made an analysis by integrating the data of the other two RCTs, ${ }^{18}$ one because it was the subgroup analyses from another RCT, ${ }^{19}$ one because it was a meta-analysis, ${ }^{20}$ and one because it was a subsequent report of another RCT. ${ }^{21}$

Three studies $^{6-8}$ met the inclusion criteria after our complete review. They all compared ADA treatment to placebo. Two studies from Europe or North America, one from Japan. 1,366 patients with moderate-to-severe UC were totally grouped at random into either ADA treatment $(n=685)$ or placebo $(n=472)$. Of these, 1,157 patients were involved in the analysis of efficacy of ADA for moderate-to-severe active UC. Two trial designs were included in these studies: induction therapy and maintenance therapy. The characteristics of the involved studies were shown in Tables 1 and 2.

\section{Methodological quality assessment of included trials}

The evaluation results of the risk of bias ware presented in Figs 1 and 2. Jadad score of three studies were greater than 3 and the quality of them were high. We carried out the analysis of all data based on the intention-to-treat principle. Because the numbers of studies was not sufficient to produce a significant analysis, the investigation on publication bias could not be hold by funnel plots.

\section{Data synthesis: efficacy for induction therapy}

The frequency of short-term clinical remission, clinical response, mucosal healing of UC treated with ADA was analyzed

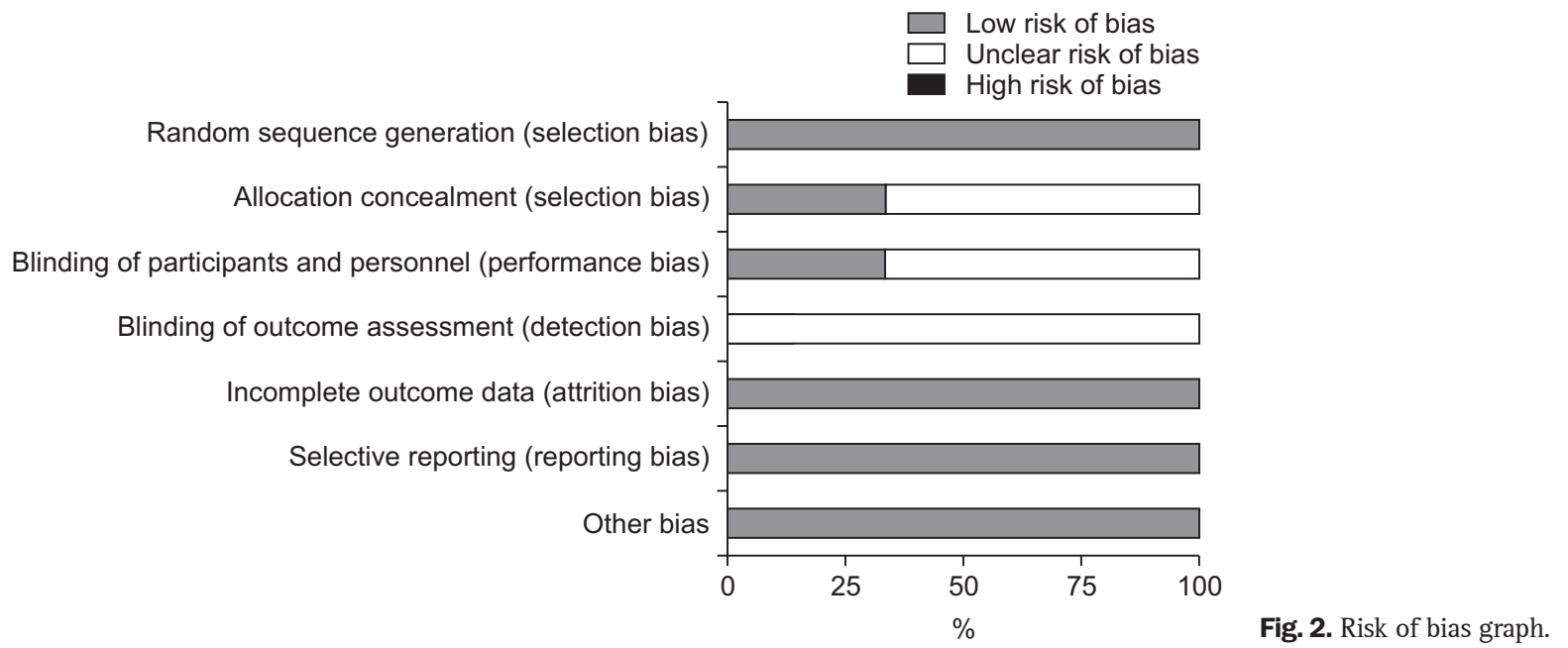


in three trials that consisted of 1,157 patients. Great heterogeneity was not found between three trials $\left(\mathrm{I}^{2}<50 \%, \mathrm{p}>0.1\right)$. Fixed-effects models were used to make a pooled analysis which showed that ADA was observably better to placebo for induction of clinical remission (RR, 1.50, 95\% CI, 1.08 to 2.09, $\mathrm{Z}=2.42$; ADA vs placebo, 14.45\% vs 9.76\%, $\mathrm{p}=0.02$ ), response ( $R R, 1.33$, 95\% CI, 1.16 to $1.52, Z=4.01$; ADA vs placebo, $50.36 \%$ vs $37.5 \%$, $\mathrm{p}<0.0001$ ), and mucosal healing (RR, 1.21, 95\% CI, 1.04 to 1.41 , $\mathrm{Z}=2.40$; ADA vs placebo, $41.75 \%$ vs $34.11 \%, \mathrm{p}=0.02$ ) (Fig. 3).

of the studies included in our analysis, two of the studies

A

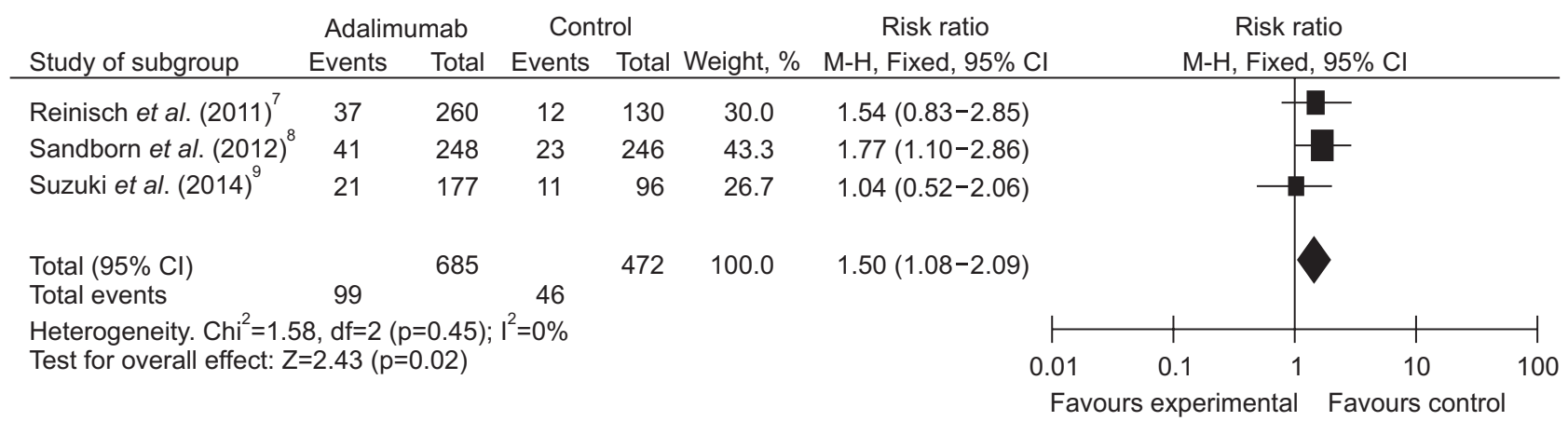

B

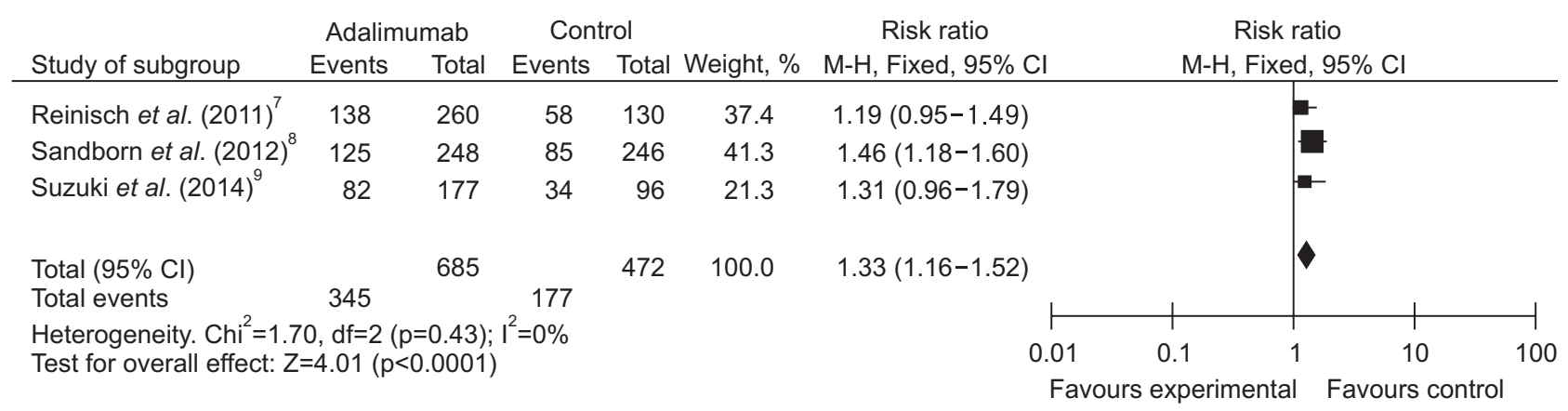

C

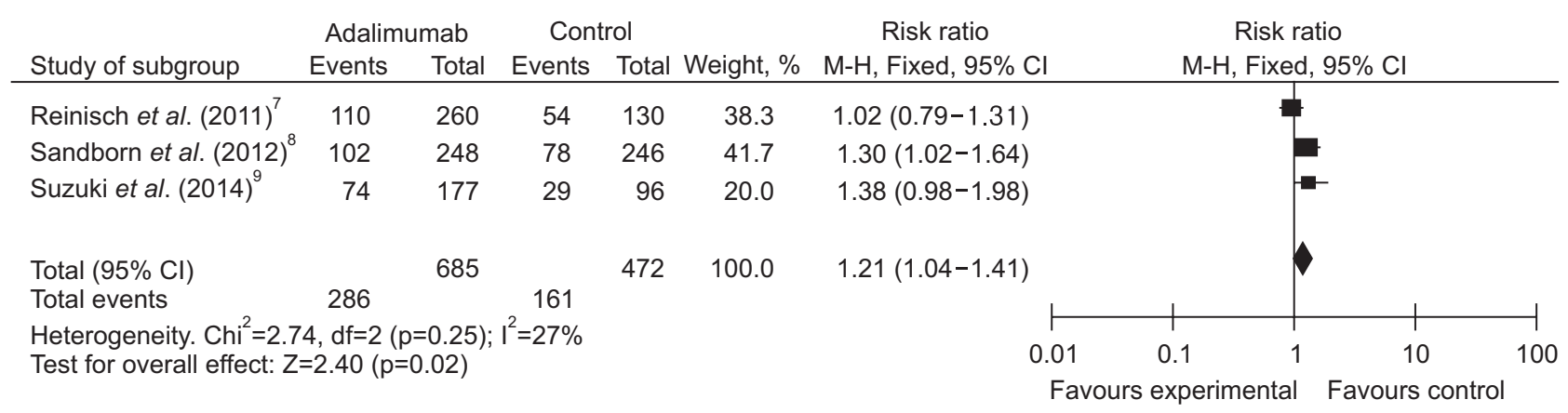

D

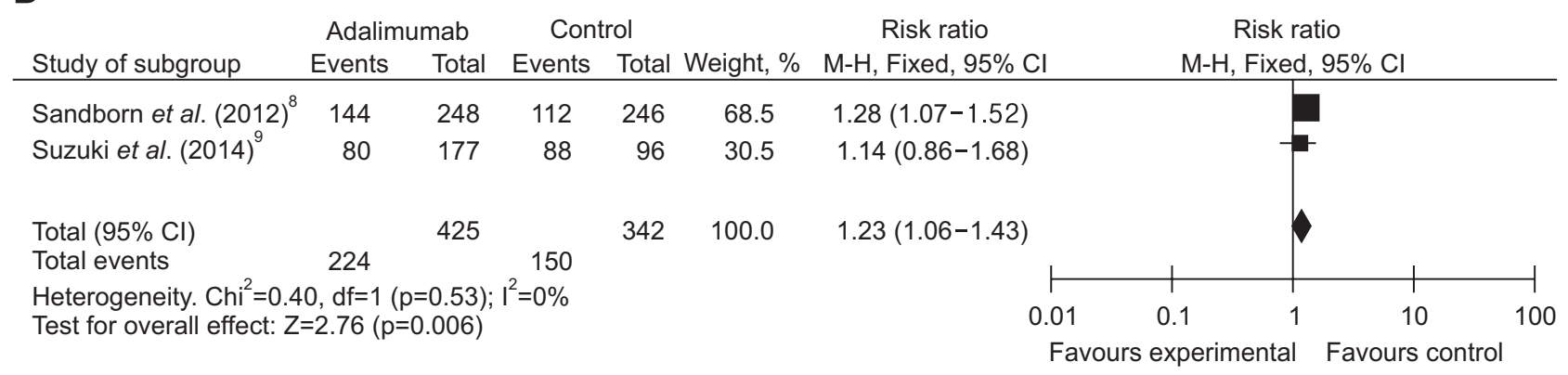

Fig. 3. Short-term results for (A) clinical remission, (B) clinical response, (C) mucosal healing, and (D) inflammatory bowel disease questionnaire (IBDQ) response in patients exposed to adalimumab versus placebo.

CI, confidence interval. 
A

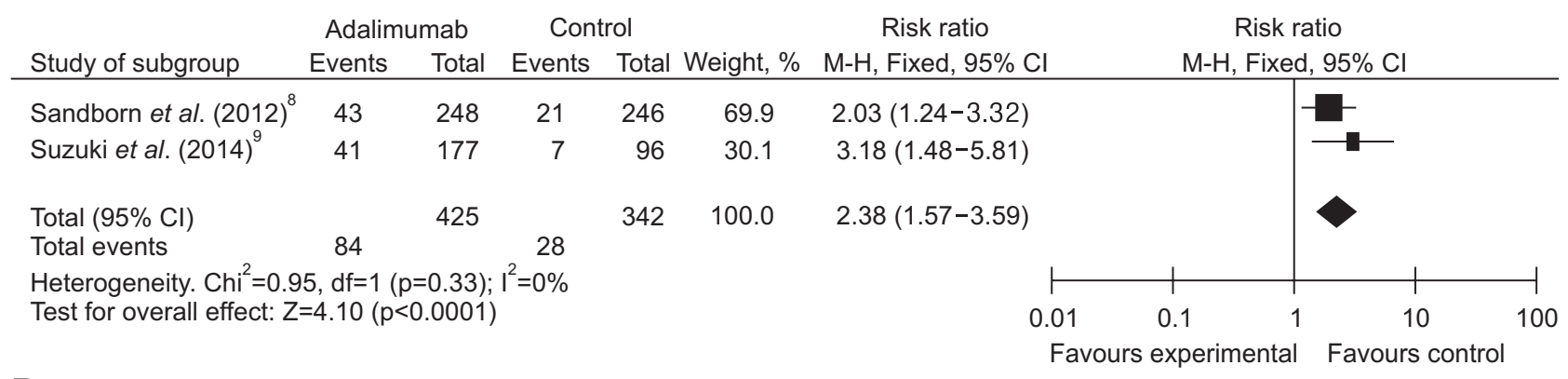

B

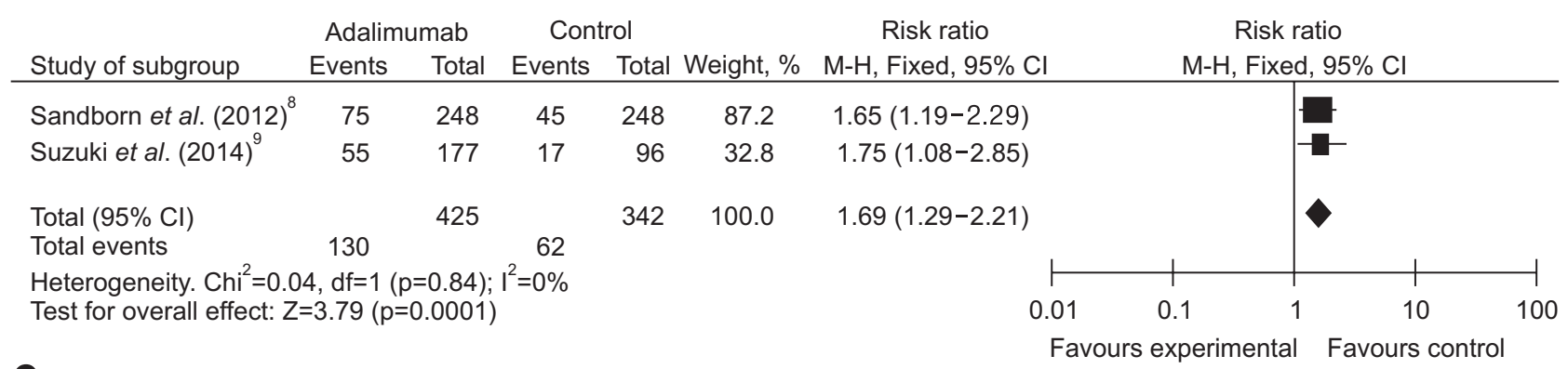

C

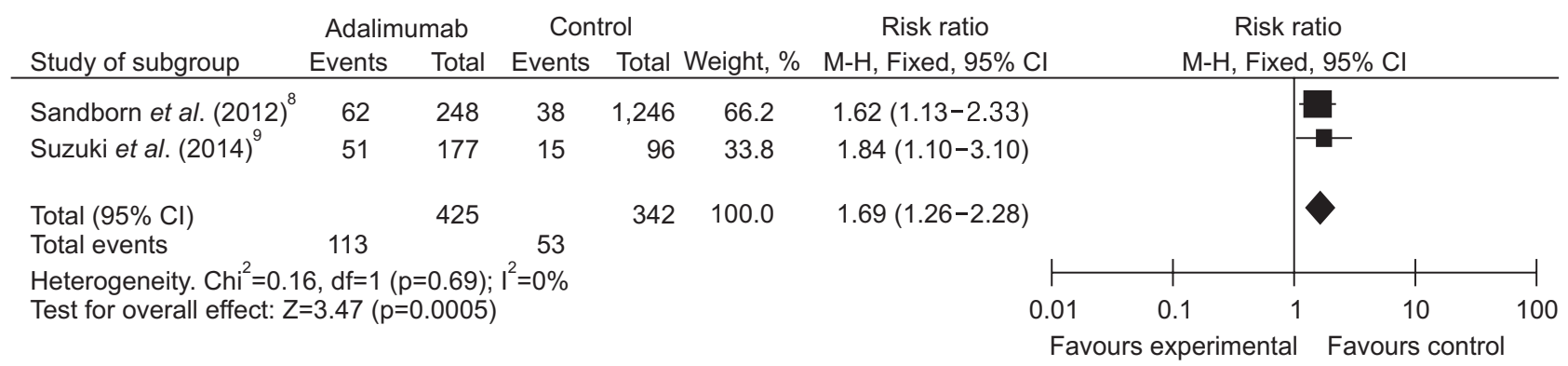

D

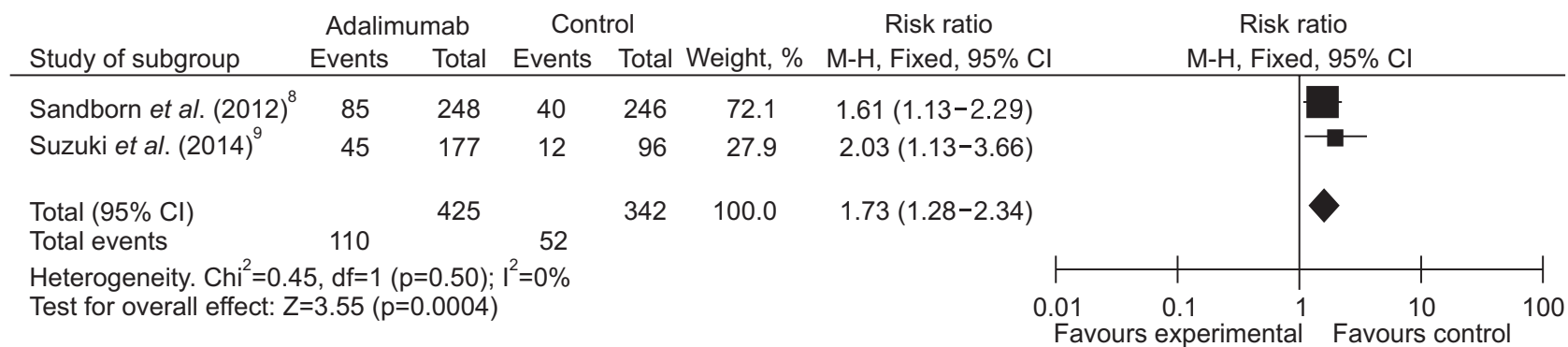

E

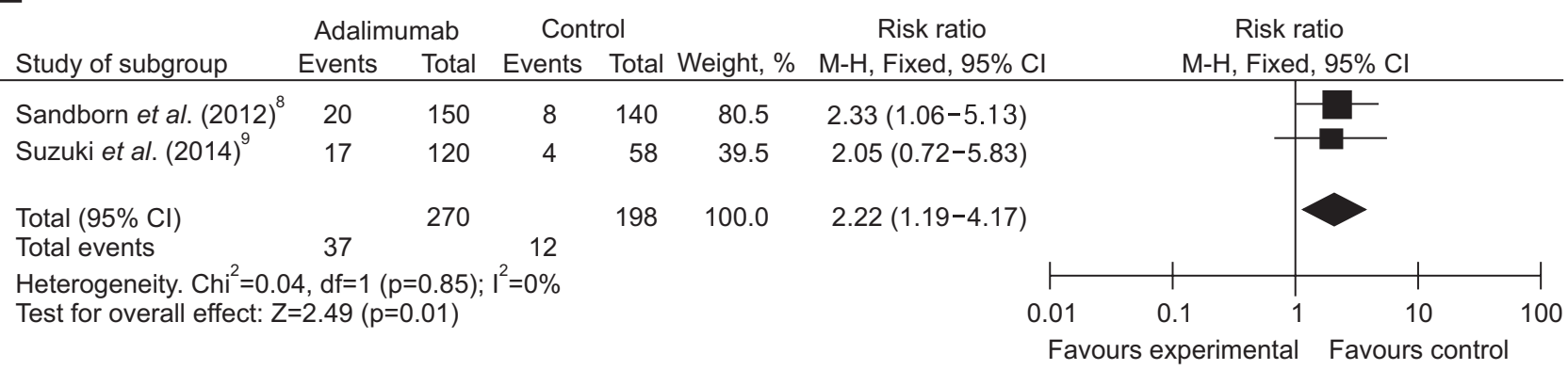

Fig. 4. Long-term results for (A) clinical remission, (B) clinical response, (C) mucosal healing, (D) inflammatory bowel disease questionnaire (IBDQ) response, and (E) steroids-free remission in patients exposed to adalimumab versus placebo.

CI, confidence interval. 
included in our analysis, composed of 767 patients, assessed the rate of response per IBDQ score of patients treated with ADA at week 8 . We have not found remarkable heterogeneity among these trials $\left(I^{2}=0 \%, p=0.53\right)$. A fixed-effects model was applied to make a pooled analysis showing that ADA was significantly superior to placebo for induction of IBDQ response (RR, 1.23, 95\% CI, 1.06 to $1.43, \mathrm{Z}=2.76$; ADA vs placebo, 52.71\% vs 43.86\%, p=0.006) (Fig. 3).

\section{Data synthesis: efficacy for maintenance therapy}

Long-term rates of clinical remission, response, mucosal healing and IBDQ response of patients treated with ADA were evaluated in two trials that consisted of 767 patients. We have detected no significant heterogeneity between these trials $\left(\mathrm{I}^{2}=0 \%, \mathrm{p}>0.1\right)$. With fixed-effects models used, a pooled analysis showed that $\mathrm{ADA}$ was significantly superior to placebo for maintenance of clinical remission (RR, 2.38, 95\% CI, 1.57 to $3.59, \mathrm{Z}=4.10$; $\mathrm{ADA}$ vs placebo, $19.76 \%$ vs $8.19 \%, \mathrm{p}<0.0001$ ), clinical response (RR, $1.69,95 \% \mathrm{CI}, 1.29$ to $2.21, \mathrm{Z}=3.79$; ADA vs placebo, $30.59 \%$ vs $23.98 \%, p=0.0001)$, mucosal healing ( $R R$, $1.69,95 \%$ CI, 1.26 to $2.68, Z=3.47$; ADA vs placebo, $26.59 \%$ vs $15.50 \%, p=0.0005)$, and IBDQ response (RR, 1.73, 95\% CI, 1.28 to $2.34, \mathrm{Z}=3.55$; ADA vs placebo, $25.88 \%$ vs $15.20 \%, \mathrm{p}=0.0004$ ) (Fig. 4).

Two trials, consisting of 468 patients, studied the frequency of steroid-free remission to determine ADA maintenance treatment efficacy. Large heterogeneity between data sources was noted $\left(\mathrm{I}^{2}=0 \%, \mathrm{p}=0.85\right)$. A total analysis applying fixed-effects models demonstrated that the percentage of patients who achieved steroid-free remission was larger in groups that received ADA

A

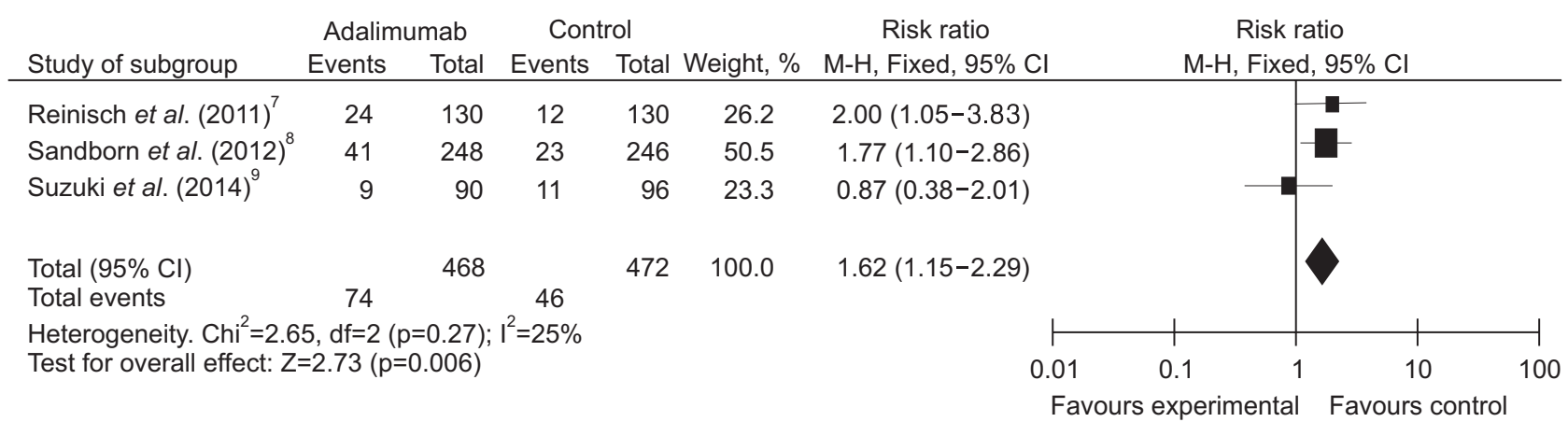

B

\begin{tabular}{|c|c|c|c|c|c|c|c|c|c|}
\hline \multirow[b]{2}{*}{ Study of subgroup } & \multicolumn{2}{|c|}{ Adalimumab } & \multicolumn{2}{|c|}{ Control } & \multirow[b]{2}{*}{ Weight, \% } & \multirow{2}{*}{$\begin{array}{c}\text { Risk ratio } \\
\mathrm{M}-\mathrm{H}, \text { Fixed, } 95 \% \mathrm{Cl} \\
\end{array}$} & \multirow{2}{*}{\multicolumn{2}{|c|}{$\begin{array}{c}\text { Risk ratio } \\
\mathrm{M}-\mathrm{H}, \text { Fixed, } 95 \% \mathrm{Cl} \\
\end{array}$}} & \\
\hline & Events & Total & Events & Total & & & & & \\
\hline Reinisch et al. (2011) ${ }^{7}$ & 71 & 130 & 58 & 130 & 32.9 & $1.22(0.96-1.57)$ & & & \\
\hline Sandborn et al. (2012) & 125 & 248 & 85 & 246 & 48.4 & $1.46(1.18-1.80)$ & & 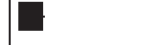 & \\
\hline Suzuki et al. (2014) & 45 & 90 & 34 & 96 & 18.7 & $1.41(1.00-1.98)$ & & - & \\
\hline Total $(95 \% \mathrm{Cl})$ & & 468 & & 472 & 100.0 & 1.37 (1.19-1.59) & & $\boldsymbol{\nabla}$ & \\
\hline Total events & 241 & & 177 & & & & & & \\
\hline $\begin{array}{l}\text { Heterogeneity. } \mathrm{Chi}^{2}=1.1 \\
\text { Test for overall effect: } \mathrm{Z}\end{array}$ & $\begin{array}{l}\text { 17, } d f=2(p \\
=4.26(p<\end{array}$ & $\begin{array}{l}=0.56) ; \\
.0001\end{array}$ & $I^{2}=0 \%$ & & & 0.0 & 0.1 & 10 & 100 \\
\hline
\end{tabular}

C

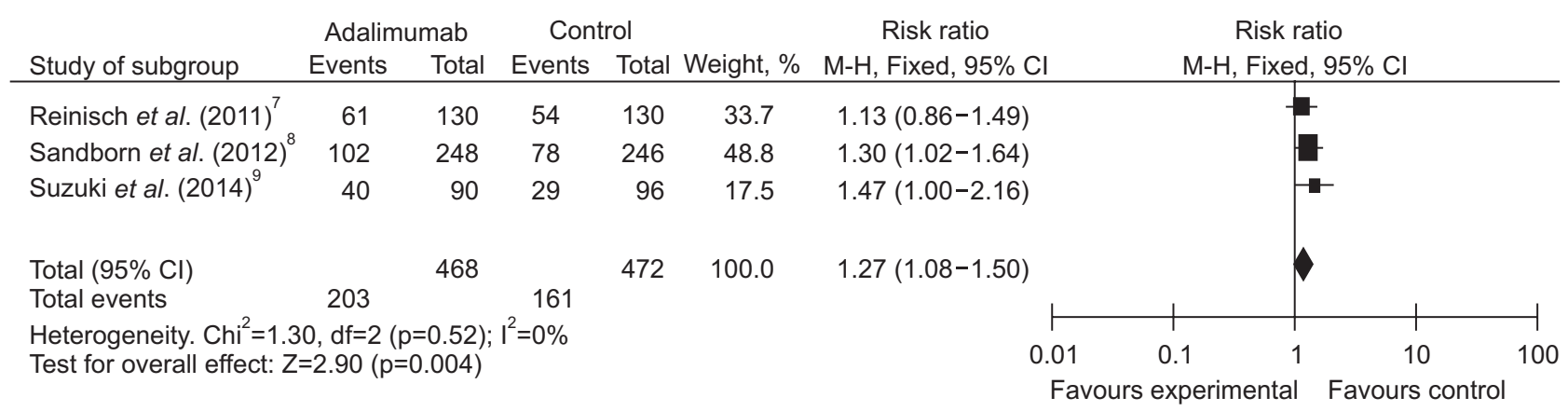

Fig. 5. Short-term results for (A) clinical remission, (B) clinical response, and (C) mucosal healing in patients exposed to adalimumab (160/80 mg at weeks $0 / 2$ ) versus placebo.

$\mathrm{CI}$, confidence interval. 
than the placebo groups (RR, 2.22, 95\% CI, 1.19 to 4.17, Z, 2.49; ADA vs placebo, $13.70 \%$ vs 6.06\%, $p=001$ ) (Fig. 4).

\section{Data synthesis: efficacy of ADA therapy with different loading doses}

Short-term efficacy in patients receiving the ADA $160 \mathrm{mg}$ at week 0 , measured by rates of clinical remission, response and mucosal healing, was studied by three trials which evaluated a total of 940 patients. No great heterogeneity was detected among the studies when the data were pooled for analysis $\left(\mathrm{I}^{2}<50 \%, \mathrm{p}>0.1\right)$. Therefore, a fixed effects model of analysis was chosen, the overall analysis revealed that ADA significantly increased the short-term efficacy compared with placebo, including clinical remission (RR, 1.62, 95\% CI, 1.15 to $2.29, \mathrm{Z}=2.73$; ADA vs placebo, 15.81\% vs 9.75\%, $p=0.006$ ), clinical response (RR, $1.37,95 \%$ CI, 1.19 to $1.59, Z=4.26$; ADA vs placebo,
51.50\% vs 37.5\%, $\mathrm{p}<0.0001)$, and mucosal healing (RR, 1.27, 95 CI, 1.08 to $1.50, \mathrm{Z}=2.90$; ADA vs placebo, $43.38 \%$ vs $34.11 \%$, $\mathrm{p}=0.004$ ) (Fig. 5).

Two trials included which consist of 443 patients, evaluated short-term rates of clinical remission, response and mucosal healing in patients assigned to receive a induction dosing of ADA $80 \mathrm{mg}$ at week 0 . Based on fixed-effects models, large differences $\left(\mathrm{I}^{2}<50 \%, \mathrm{p}>0.1\right)$ was not detected in rates of clinical remission (RR, 1.14, 95\% CI, 0.67 to $1.94, \mathrm{Z}=0.48$; ADA vs placebo, $11.68 \%$ vs $10.18 \%, p=0.63)$, clinical response $(R R$, 1.17, 95\% CI, 0.95 to $1.44, \mathrm{Z}=1.49$; ADA vs placebo, $47.92 \%$ vs $40.71 \%, \mathrm{p}=0.14)$, and mucosal healing (RR, 1.04, 95\% CI, 0.82 to $1.32, \mathrm{Z}=0.30$; $\mathrm{ADA}$ vs placebo, $38.25 \%$ vs $36.73 \%, \mathrm{p}=0.76$ ) between the ADA and placebo treatment (Fig. 6).

Besides, the week 52 clinical remission rate of patients receiving the induction dose of ADA $160 \mathrm{mg}$ was studied in two trials

A

\begin{tabular}{|c|c|c|c|c|c|c|c|c|}
\hline \multirow[b]{2}{*}{ Study of subgroup } & \multicolumn{2}{|c|}{ Adalimumab } & \multicolumn{2}{|c|}{ Control } & \multirow[b]{2}{*}{ Weight, \% } & Risk ratio & Risk ratio & \\
\hline & Events & Total & Events & Total & & $\mathrm{M}-\mathrm{H}$, Fixed, $95 \% \mathrm{Cl}$ & & \\
\hline Reinisch et al. (2011) & 13 & 130 & 12 & 130 & 53.4 & $1.08(0.51-2.28)$ & & \\
\hline Suzuki et al. $(2014)^{9}$ & 12 & 87 & 11 & 96 & 46.8 & $1.20(0.56-2.59)$ & & \\
\hline $\begin{array}{l}\text { Total }(95 \% \mathrm{Cl}) \\
\text { Total events }\end{array}$ & 25 & 217 & 23 & 226 & 100.0 & $1.14(0.67-1.94)$ & & \\
\hline Heterogeneity. $\mathrm{Chi}^{2}=0$ & $4, d f=1$ & $0.85)$ & $1^{2}=0 \%$ & & & & & \\
\hline Test for overall effect: & $=0.48(p$ & .63) & & & & 0.01 & 0.1 & 100 \\
\hline
\end{tabular}

B

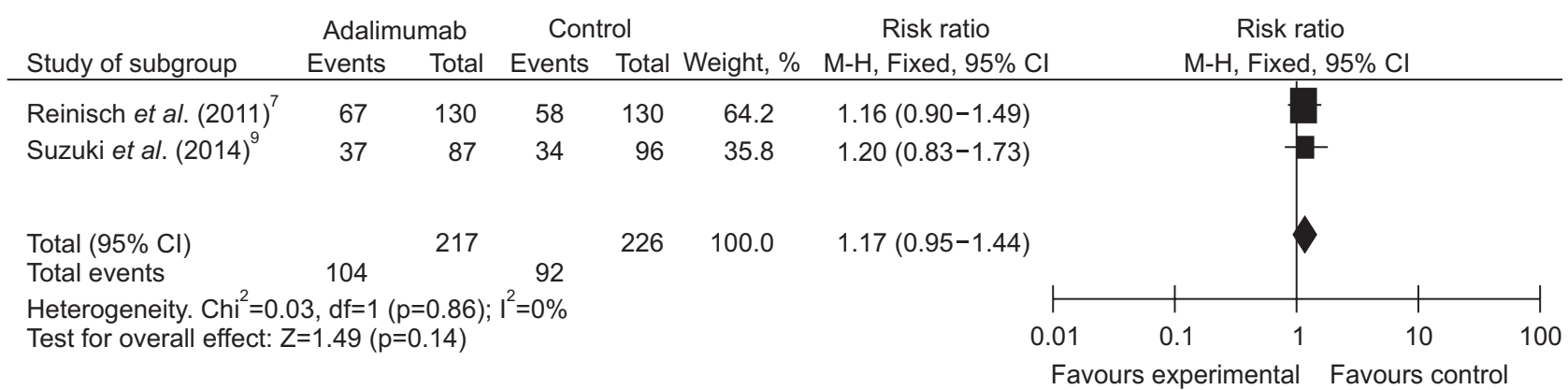

C

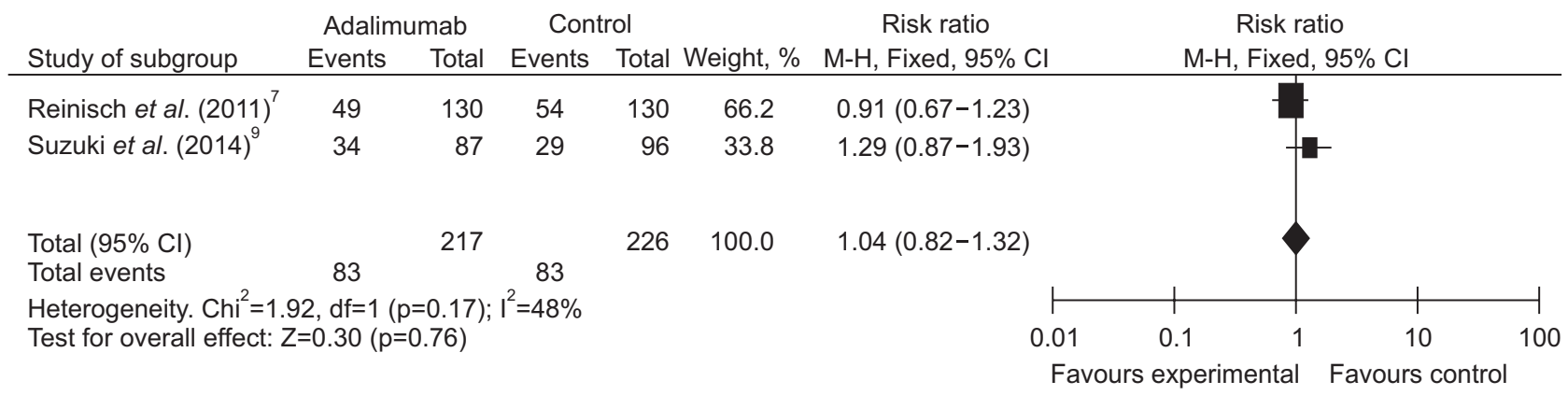

Fig. 6. Short-term results for (A) clinical remission, (B) clinical response, and (C) mucosal healing in patients exposed to adalimumab (80/40 mg at weeks $0 / 2$ ) versus placebo. $\mathrm{CI}$, confidence interval. 
that consisted of 680 patients. No large differences was found among these trials $\left(I^{2}=0 \%, p=0.33\right)$. With fixed-effects models, we made a pooled analysis showing that ADA 160/80 mg significantly better to placebo in terms of maintaining clinical remission ( $R R, 2.20 ; 95 \% \mathrm{CI}, 1.45$ to $3.36 ; \mathrm{Z}=3.68 ; \mathrm{p}=0.0002)$ at week 52.

A

\begin{tabular}{|c|c|c|c|c|c|c|c|c|}
\hline \multirow[b]{2}{*}{ Study of subgroup } & \multicolumn{2}{|c|}{ Adalimumab } & \multicolumn{2}{|c|}{ Control } & \multirow[b]{2}{*}{ Weight, \% } & \multirow{2}{*}{$\begin{array}{c}\text { Risk ratio } \\
\mathrm{M}-\mathrm{H}, \text { Fixed, } 95 \% \mathrm{Cl} \\
\end{array}$} & \multirow{2}{*}{$\begin{array}{c}\text { Risk ratio } \\
\mathrm{M}-\mathrm{H} \text {, Fixed, } 95 \% \mathrm{Cl}\end{array}$} & \\
\hline & Events & Total & Events & Total & & & & \\
\hline Reinisch et al. (2011) ${ }^{7}$ & 21 & 145 & 8 & 89 & 49.0 & $1.61(0.75-3.48)$ & & \\
\hline Suzuki et al. (2014) & 15 & 120 & 10 & 58 & 51.0 & $0.72(0.35-1.51)$ & & \\
\hline $\begin{array}{l}\text { Total }(95 \% \mathrm{Cl}) \\
\text { Total events }\end{array}$ & 36 & 265 & 18 & 147 & 100.0 & $1.07(0.49-2.35)$ & & \\
\hline \multirow{2}{*}{\multicolumn{6}{|c|}{$\begin{array}{l}\text { Heterogeneity. Tau }{ }^{2}=0.17, \mathrm{Chi}^{2}=2.18, \mathrm{df}=1(p=0.14) ;\left.\right|^{2}=54 \% \\
\text { Test for overall effect: } Z=0.17(p=0.86)\end{array}$}} & $\longmapsto$ & + & \\
\hline & & & & & & 0.01 & 0.1 & 100 \\
\hline
\end{tabular}

B

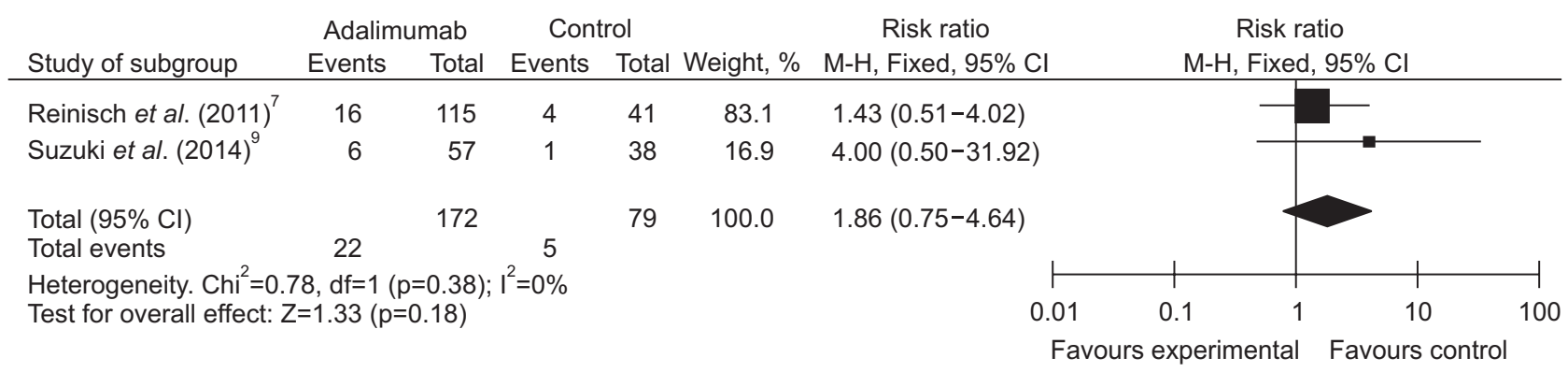

Fig. 7. Short-term results for clinical remission in the (A) steroids and (B) no steroids subgroups exposed to adalimumab versus placebo. CI, confidence interval.

A

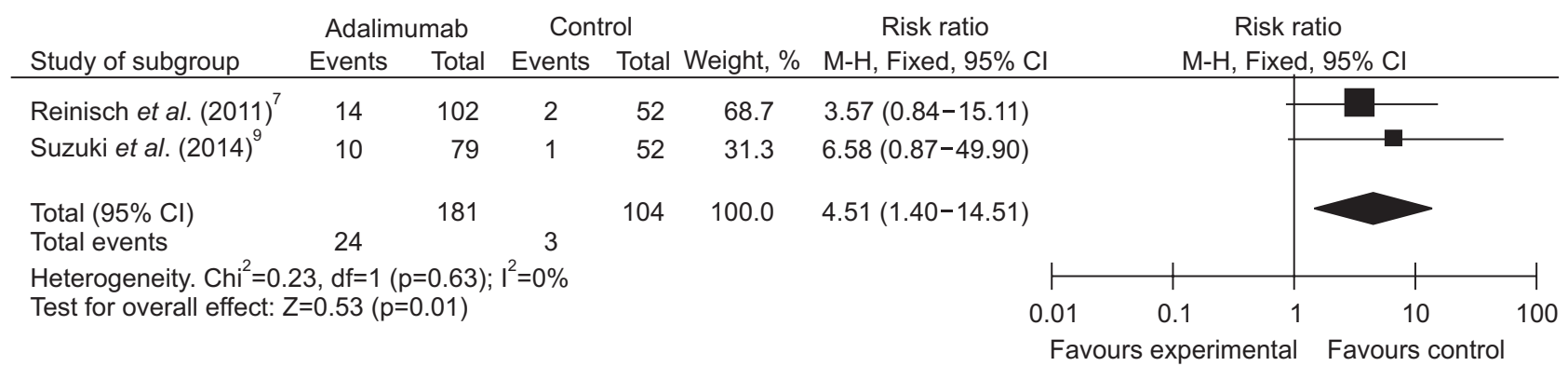

B

\begin{tabular}{|c|c|c|c|c|c|c|c|c|}
\hline \multirow[b]{2}{*}{ Study of subgroup } & \multicolumn{2}{|c|}{ Adalimumab } & \multicolumn{2}{|c|}{ Control } & \multirow[b]{2}{*}{ Weight, \% } & \multirow{2}{*}{$\begin{array}{c}\text { Risk ratio } \\
\mathrm{M}-\mathrm{H} \text {, Fixed, } 95 \% \mathrm{Cl} \\
\end{array}$} & \multirow{2}{*}{$\begin{array}{c}\text { Risk ratio } \\
\text { M-H, Fixed, } 95 \% \mathrm{Cl} \\
\end{array}$} & \\
\hline & Events & Total & Events & Total & & & & \\
\hline Reinisch et al. (2011) ${ }^{7}$ & 23 & 158 & 10 & 78 & 52.4 & $1.14(0.57-2.27)$ & & \\
\hline Suzuki et al. (2014) & 11 & 98 & 10 & 44 & 47.6 & $0.49(0.23-1.08)$ & & \\
\hline $\begin{array}{l}\text { Total }(95 \% \mathrm{Cl}) \\
\text { Total events }\end{array}$ & 34 & 256 & 20 & 122 & 100.0 & $0.76(0.34-1.73)$ & & \\
\hline $\begin{array}{l}\text { Heterogeneity. } \operatorname{Tau}^{2}=0 \text {. } \\
\text { Test for overall effect: }\end{array}$ & $\begin{array}{l}21 ; \mathrm{Chi}^{2}= \\
=0.65(\mathrm{p}=\end{array}$ & $\begin{array}{l}47, \mathrm{df}= \\
.52)\end{array}$ & $1(p=0.1$ & )$; 1^{2}=5$ & & 0.01 & 0.1 & 100 \\
\hline
\end{tabular}

Fig. 8. Short-term results for clinical remission in the (A) immunomodulators (IMM) and (B) no IMM subgroups exposed to adalimumab versus placebo.

CI, confidence interval. 


\section{Data synthesis: short-term efficacy of ADA therapy by baseline medications}

Short-term treatment effect of ADA in terms of clinical remission by baseline corticosteroids/IMM was evaluated in two studies consisting of 663 patients. A random effects model of analysis was applied due to remarkable heterogeneity $\left(\mathrm{I}^{2}=54 \%\right.$, $\mathrm{p}=0.14$ ); the pooled analysis revealed that week 8 clinical remission rates were equivalent between the ADA and placebo groups in corticosteroids subgroup (RR, 1.10, 95\% CI, 0.65 to $1.86, \mathrm{Z}=0.36$; $\mathrm{ADA}$ vs placebo, $13.58 \%$ vs $12.24 \%, \mathrm{p}=0.72$ ). In no corticosteroids subgroup, we applied the fixed effects model because of no large heterogeneity $\left(\mathrm{I}^{2}=0 \%, \mathrm{p}=0.38\right)$ and found that clinical remission rate were equivalent between the ADA and placebo groups in no corticosteroids subgroup at week 8 (RR, $1.86,95 \%$ CI, 0.75 to $4.64, \mathrm{Z}=1.33$; ADA vs placebo, $12.80 \%$ vs $10.18 \%, p=0.18$ ) (Fig. 7). In the IMM subgroup, a fixed effects model was used after detecting no great heterogeneity among the data sources, and we found a significant higher week 8 clinical remission rate in patients receiving ADA versus placebo (RR, 4.51, 95\% CI, 1.40 to $14.51, \mathrm{Z}=0.53$; ADA vs placebo, $13.26 \%$ vs $2.88 \%, p=0.01$ ); whereas, since significant heterogeneity detected in rates of clinical remission $\left(\mathrm{I}^{2}=59 \%, \mathrm{p}=0.12\right)$, we used the random effects model and found that the week 8 clinical remission rate in $\mathrm{ADA}$ group was equal with that in placebo group when not receiving the IMM at the baseline (RR, $0.81,95 \%$ CI, 0.49 to $1.35, \mathrm{Z}=0.81$; ADA vs placebo, $13.27 \%$ vs $10.18 \%, \mathrm{p}=0.42$ ) (Fig. 8).

\section{Data synthesis: safety of ADA}

The possible side effects of ADA include infections, injection site reactions, allergic reaction, opportunistic infection, congestive heart failure, lupus-like syndrome and so on. The morbidity of any adverse events (AEs) and serious adverse events (SAEs) were analyzed to assess the safety of ADA in two trials, consisting of 610 patients in the ADA groups and 483 patients receiv-

A

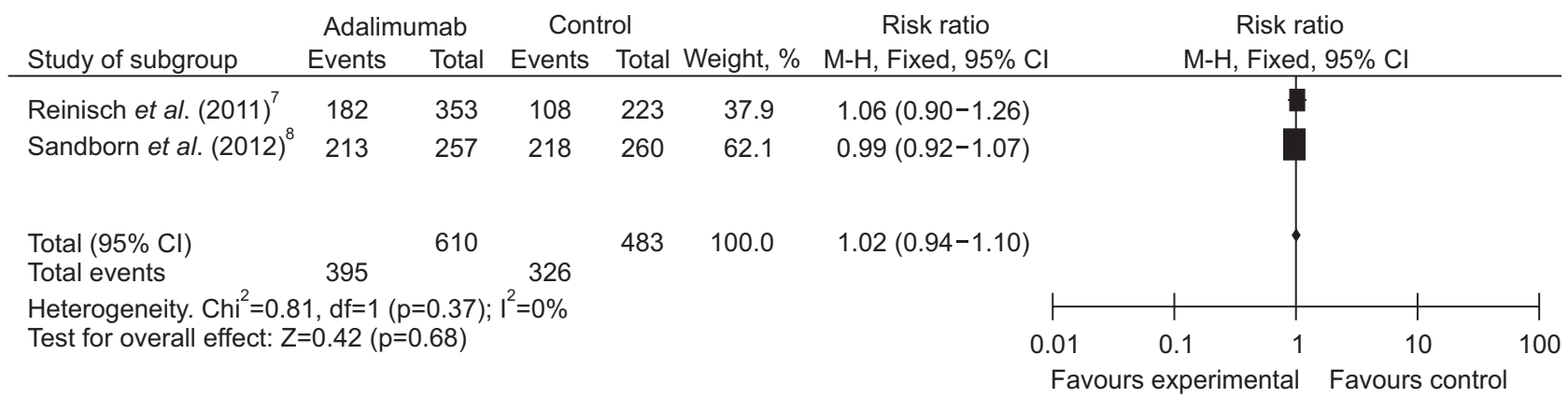

B

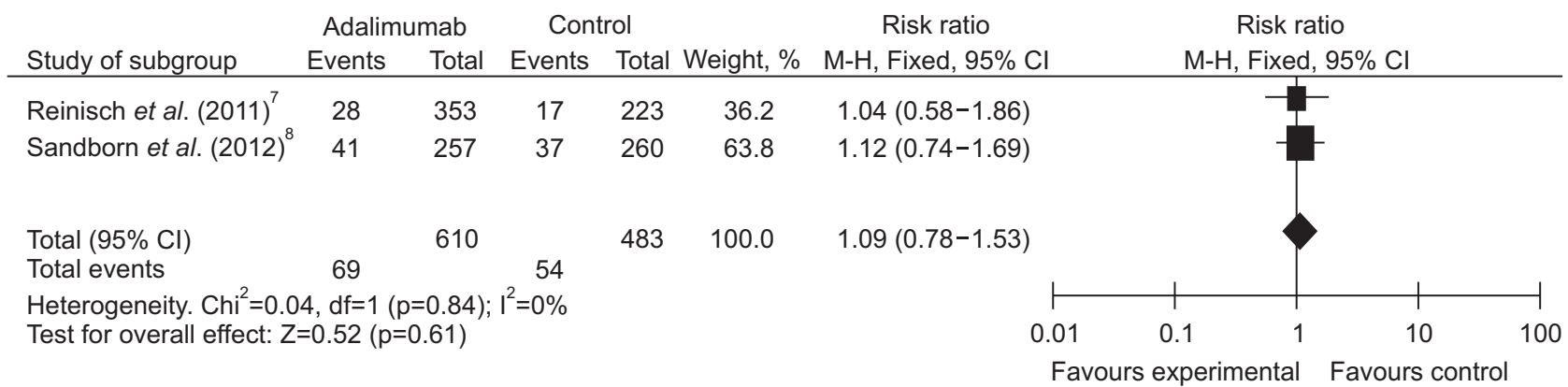

C

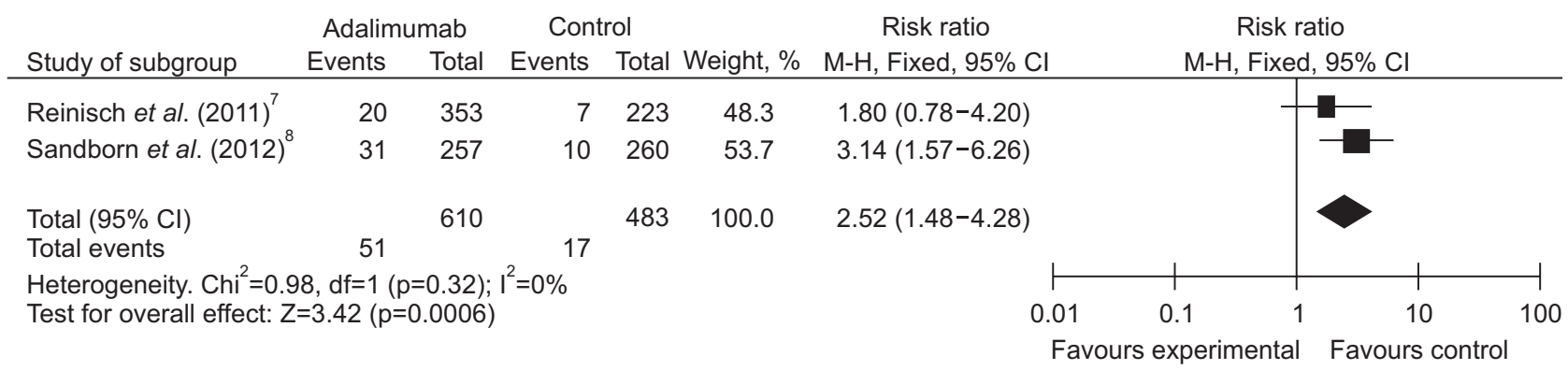

Fig. 9. Outcomes for (A) side effects, (B) serious side effects, and (C) injection-site reaction in patients exposed to adalimumab versus placebo. CI, confidence interval. 
Table 3. Sensitivity Analysis

\begin{tabular}{|c|c|c|c|c|}
\hline Statistical index (rate) & No. of included study & Statistical method & RR $(95 \% \mathrm{CI})$ & $\mathrm{p}$-value \\
\hline \multirow[t]{2}{*}{ Short-term clinical remission } & 3 & Fixed & $1.50(1.08-2.09)$ & 0.0200 \\
\hline & & Random & $1.50(1.08-2.09)$ & 0.0200 \\
\hline \multirow[t]{2}{*}{ Short-term clinical response } & 3 & Fixed & $1.33(1.16-1.52)$ & $<0.0001$ \\
\hline & & Random & $1.32(1.15-1.52)$ & $<0.0001$ \\
\hline \multirow[t]{2}{*}{ Short-term mucosal healing } & 3 & Fixed & $1.21(1.04-1.41)$ & 0.0200 \\
\hline & & Random & $1.20(1.00-1.44)$ & 0.0500 \\
\hline \multirow[t]{2}{*}{ Short-term IBDQ response } & 2 & Fixed & $1.23(1.06-1.43)$ & 0.0060 \\
\hline & & Random & $1.24(1.07-1.44)$ & 0.0050 \\
\hline \multirow[t]{2}{*}{ Long-term clinical remission } & 2 & Fixed & $2.38(1.57-3.59)$ & $<0.0001$ \\
\hline & & Random & $2.32(1.53-3.50)$ & $<0.0001$ \\
\hline \multirow[t]{2}{*}{ Long-term clinical response } & 2 & Fixed & $1.69(1.69-2.21)$ & 0.0001 \\
\hline & & Random & $1.68(1.29-2.21)$ & 0.0002 \\
\hline \multirow[t]{2}{*}{ Long-term mucosal healing } & 2 & Fixed & $1.69(1.26-2.28)$ & 0.0005 \\
\hline & & Random & $1.69(1.25-2.28)$ & 0.0006 \\
\hline \multirow[t]{2}{*}{ Long-term IBDQ response } & 2 & Fixed & $1.73(1.28-2.34)$ & 0.0004 \\
\hline & & Random & $1.71(1.27-2.32)$ & 0.0005 \\
\hline \multirow[t]{2}{*}{ Long-term steroid-free remission } & 2 & Fixed & $2.22(1.19-4.17)$ & 0.0100 \\
\hline & & Random & $2.23(1.19-4.18)$ & 0.0100 \\
\hline \multirow[t]{2}{*}{ Short-term clinical remission, $160 / 80 \mathrm{mg} \mathrm{0/2} \mathrm{wk}$} & 3 & Fixed & $1.62(1.15-2.29)$ & 0.0060 \\
\hline & & Random & $1.58(1.05-2.40)$ & 0.0300 \\
\hline \multirow[t]{2}{*}{ Short-term clinical response, $160 / 80 \mathrm{mg} \mathrm{0/2} \mathrm{wk}$} & 3 & Fixed & $1.37(1.19-1.59)$ & $<0.0001$ \\
\hline & & Random & $1.36(1.18-1.58)$ & $<0.0001$ \\
\hline \multirow[t]{2}{*}{ Short-term mucosal healing, $160 / 80 \mathrm{mg} \mathrm{0/2} \mathrm{wk}$} & 3 & Fixed & $1.27(1.08-1.50)$ & 0.0004 \\
\hline & & Random & $1.26(1.08-1.49)$ & 0.0005 \\
\hline \multirow[t]{2}{*}{ Short-term clinical remission, $80 / 40 \mathrm{mg} 0 / 2 \mathrm{wk}$} & 2 & Fixed & $1.14(0.67-1.94)$ & 0.6300 \\
\hline & & Random & $1.14(0.67-1.95)$ & 0.6300 \\
\hline \multirow[t]{2}{*}{ Short-term clinical response, $80 / 40 \mathrm{mg} \mathrm{0/2} \mathrm{wk}$} & 2 & Fixed & $1.17(0.95-1.44)$ & 0.1400 \\
\hline & & Random & $1.17(0.95-1.44)$ & 0.1400 \\
\hline \multirow[t]{2}{*}{ Short-term mucosal healing, $80 / 40 \mathrm{mg} 0 / 2 \mathrm{wk}$} & 2 & Fixed & $1.04(0.82-1.32)$ & 0.7600 \\
\hline & & Random & $1.06(0.75-1.49)$ & 0.7600 \\
\hline \multirow[t]{2}{*}{ Long-term clinical remission, $160 / 80 \mathrm{mg} \mathrm{0/2} \mathrm{wk}$} & 2 & Fixed & $2.20(1.45-3.36)$ & 0.0002 \\
\hline & & Random & $2.20(1.44-3.35)$ & 0.0003 \\
\hline \multirow[t]{2}{*}{ Short-term clinical remission (baseline CS) } & 2 & Fixed & $1.10(0.65-1.86)$ & 0.7200 \\
\hline & & Random & $1.07(0.49-2.35)$ & 0.8600 \\
\hline \multirow[t]{2}{*}{ Short-term clinical remission (baseline no CS) } & 2 & Fixed & $1.86(0.75-4.64)$ & 0.1800 \\
\hline & & Random & $1.75(0.69-4.43)$ & 0.2400 \\
\hline \multirow[t]{2}{*}{ Short-term clinical remission (baseline IMM) } & 2 & Fixed & $4.51(1.40-14.51)$ & 0.0100 \\
\hline & & Random & $4.39(1.35-14.21)$ & 0.0100 \\
\hline \multirow[t]{2}{*}{ Short-term clinical remission (baseline no IMM) } & 2 & Fixed & $0.81(0.49-1.35)$ & 0.4200 \\
\hline & & Random & $0.76(0.34-1.73)$ & 0.5200 \\
\hline \multirow[t]{2}{*}{ Adverse event } & 2 & Fixed & $1.02(0.94-1.10)$ & 0.6800 \\
\hline & & Random & $1.00(0.93-1.07)$ & 0.9700 \\
\hline \multirow[t]{2}{*}{ Serious adverse event } & 2 & Fixed & $1.09(0.78-1.53)$ & 0.6100 \\
\hline & & Random & $1.09(0.78-1.53)$ & 0.6000 \\
\hline \multirow[t]{2}{*}{ Injection-site reaction } & 2 & Fixed & $2.52(1.48-4.28)$ & 0.0006 \\
\hline & & Random & $2.51(1.47-4.29)$ & 0.0007 \\
\hline
\end{tabular}

RR, relative risk; IBDQ, inflammatory bowel disease questionnaire; CS, corticosteroid; IMM, immunomodulators. 
ing placebo therapy. In these trials, the frequency of AEs was $64.8 \%$ in the ADA group, and 67.5\% in the placebo group. No significant heterogeneity was detected among the studies when the data were pooled for analysis. Fixed effects models were adopted to make an overall analysis showed the occurrence of AEs (RR, 1.02; 95\% CI, 0.94 to $1.10 ; \mathrm{Z}=0.42 ; \mathrm{p}=0.68$ ) and severe adverse reactions ( $R R, 1.09$; 95\% CI, 0.78 to 1.53 ; $p=0.61$ ) were equivalent between the ADA-treated group and placebo group (Fig. 9).

Two papers reported injection site reaction-related AE. Heterogeneity test indicated $\mathrm{I}^{2}=0 \%$ and $\mathrm{p}=0.32$, demonstrating homogeneity of include studies. Fixed effects model was applied accordingly, and the RR value was 2.52 (95\% CI, 1.48 to 4.28 ; $\mathrm{p}=0.0006$ ). Based on the result, ADA seems to increase the risk of injection site reaction-related AEs (Fig. 9).

\section{Sensitivity analysis}

Sensitivity analysis is an analytic method that can be used to determine sensitivity of a study result or how it will change a meta-analysis or systematic review, which can access the strength of merge results due to uncertainty about data and usage. In this article, we conducted the sensitivity analysis by transforming effects models to analyze and compare these two results separately (Table 3 ).

\section{DISCUSSION}

The management of difficult-to-treat UC presents a special challenge in the clinical practice. Even though some UC received the optimal conventional medications, they have disease relapse frequently and have difficulties in avoiding a surgery eventually. ${ }^{22}$ With the introduction of biological drugs, the therapy of UC has changed over the recent decade. ${ }^{23}$ Emergency of new type of biological agents increases treatments options for many UC patients. Biological agents, especially anti-TNF antibodies, have been approved by the FDA for treating some kinds of autoimmune diseases and achieved the desired effect. ${ }^{24-27}$ Now the most widely used anti-TNF- $\alpha$ antibodies in clinic is IFX. However, its high immunogenicity and high rate of AE, as well as inconvenient mode of administration, limit its applications to some extent. With the similar mode of action with IFX, ADA has been used more and more widely in clinical practice, due to low adverse reactions and its easy administration by subcutaneous injection. Therefore, ADA treatment might be considered for moderately to severely active UC who were not able to respond to or were intolerant to IFX.

According to our knowledge, it is a meta-analysis of the available published randomized placebo-controlled trials to examine the efficacy and safety of ADA in the treatment of UC. Whether it was the primary or second efficacy end point, outcomes of these studies all suggested that ADA therapy was statistically superior to a placebo for treatment of UC patients.
Therefore, ADA therapy is effective in inducing and maintaining clinical remission for patients who are unresponsive to conventional therapy. This may be related to the mechanism of action of TNF antagonists, which can prevent excessive inflammatory reaction by binding to the soluble and transmembrane forms of this cytokine. ${ }^{28}$

As to the induction therapy, two induction therapies (160/80 $\mathrm{mg}$, week 0/2 and 80/40 mg, week 0/2) of ADA were adopted in the first and third RCT, with one (80/40 mg, week 0/2) adopted in the second RCT. We analyzed the efficiency of different induction doses of ADA for patients with moderate-to-severe UC respectively and detected that a statistically larger percentage of patients receiving ADA 160/80 mg achieved clinical remission, clinical response, mucosal healing at week 8 compared with placebo. Whereas two trials showed that rates of clinical remission, clinical response, mucosal healing at week 8 were similar between those who received ADA 80/40 mg induction dose and placebo-received patients. In addition, a significantly higher clinical remission rate at week 52 was achieved in patients who were treated with 160/80/40 mg every other week (EOW) than in placebo-received patients in this study. ULTRA (Ulcerative Colitis Long-Term Remission and Maintenance with Adalimumab) 2 suggested that 1-year results were superior in week 8 responders compared with the total intent-to-treat population. And Reinisch et $a l^{21}$ also observed a significantly lower rate of dose escalation in patients who were given the 160/80 induction dose $(22.5 \%)$ compared with those who were treated with the $80 / 40 \mathrm{mg}$ induction dose (30.0\%) or placebo patients who had received no induction dose (37.7\%). All the above analysis, supports initiating ADA therapy with induction dosing of 160/80 mg.

Given that the plateau of dose-response curve was not yet achieved in ULTRA 1, and the induction dose of ADA 80/40 mg were detected effective to a considerable proportion of patients who are moderate-to-severe UC in our clinical practice, which suggests that more comprehensive clinical trial protocols could be made that involves multiple aspects including age, race, region, and so on, and group participants could be placed into more treatment arms to explore the optimized induction dosing for treatment of UC under different conditions.

As to the maintenance therapy, it is unknown whether the ADA treatment of $40 \mathrm{mg}$ weekly maintenance dose might have greater efficacy when comparing another maintenance treatment of $40 \mathrm{mg}$ EOW. However, differences were not observed between $40 \mathrm{mg}$ EOW and $40 \mathrm{mg}$ weekly in a dosing-finding maintenance trial with $\mathrm{ADA}$ in $\mathrm{CD}^{29}$

What is mentioned above was consistent with the current guideline from the FDA about the application of ADA generally: an induction region of hypodermic ADA $160 \mathrm{mg}$ at week 0, $80 \mathrm{mg}$ at week 2, and $40 \mathrm{mg}$ EOW would be effective therapy for UC. Patients should discontinue using this study drug, if patients could not manage to achieve clinical remission at week 8 
or $12 .^{30}$

Short-term efficacy of ADA by baseline concomitant medications was analyzed in two studies, which concluded that $\mathrm{ADA}$ was superior to placebo for UC who were receiving IMM at baseline in the induction phase, whereas the similar week 8 remission rates were observed in the $\mathrm{ADA}$ and placebo groups who were not receiving baseline IMM. Besides, we failed to observe an increased week 8 remission rates in the ADA arms comparing with the placebo group, regardless of receiving steroids or not at baseline. With great and unacceptable heterogeneity between the involved studies in which UC patients applied steroids or IMM at baseline during the process, it may be because the participants of all studies come from different areas of the world. Grouping on regions was not possible due to few studies, which prompt that more RCTs can be carried out to attain diversification of studies from evidence based medicine. As mentioned above, ADA seems more effective in achieving shortterm efficacy by baseline IMM medication, that is, combination IMM therapy may enhance effectiveness of ADA. This might be because IMM are able to reduce the production of anti-ADA antibodies and maintain a relatively high plasma concentration to a certain extent. Caution should be interpreted this analysis in further, because the included studies are few and the numbers of patients in each subgroup is small.

As regards the safety, we found the risks of AEs and serious side effects were comparable among the ADA group and the placebo group. Overall, SAEs happened in 11.18\% of UC in the placebo arms and $11.31 \%$ of patients in the ADA groups. Although the rate of injection site reaction was statistically higher in the ADA arms compared with the control groups, almost all of them were mild and none required discontinuation of study drug therapy. On the whole, ADA treatment was well tolerated and the total safety profile of ADA was similar with that of placebo. However, we need to interpret the data with caution because of several limitations: patients seen in clinical practice might not be represented by patients in clinical trials; the sample size is limited in the current studies; and duration of followup might be too short for some serious events to happen.

There remain several limitations in this study. Firstly, the period of patient following-up in the analyzed trials have only two cases, 8 weeks and 52 weeks. Secondly, the cotherapy regimens of ADA at baseline differed among these included trials. Thirdly, due to placebo with indefinite components, it was unknown if the placebo of three trials would cause different impacts on UC. And a part of participants in the placebo arms have been administered any other TNF- $\alpha$ antagonist therapy before, thus the efficacy of placebo in these patients could be considered. Fourthly, although the pool data about of ULTRA 1, 2 and the open-label extension ULTRA 3 has been presented recently, ${ }^{31}$ which shows that prolonged ADA therapy for 4 long years has an excellent tolerability and is effective to patients who were diagnosed as moderate-to-severe UC in maintaining clinical remission and mucosal healing, it cannot be included because of lacking a control group during the maintenance phase from week 52 to week 208. So it is difficult to evaluate systemically the maintenance outcomes of longer than 52 weeks among patients who were diagnosed as moderate-to-severe active UC who have response to induction therapy with ADA. Future studies should be carried out further to access the efficacy and safety of ADA in UC for longer time. Fifthly, all the involved studies are clinical pharmaceutical trials in which contributors have been strictly screened. However, patients in clinical practice might be more inhomogeneous and subject to different circumstances. Additionally, publication bias was of concern and we should interpret our outcomes with care due to the small number of researches that met our inclusion criteria. All examples of variability mentioned above could affect the outcomes drawn from our analysis. Despite these limitations, the meta-analysis findings are strengthened by the fact that all studies included were of high quality (Jadad score $\geq 3$ ).

In summary, the UC treatment field has been updated in this meta-analysis and the conclusion has been suggested that $\mathrm{ADA}$ was superior to placebo. We came up to the conclusion on basis of increasing the achievement of clinical remission, response, mucosal healing and IBDQ response at weeks 8 and 52 without significant, severe side effects. So, in screened participants with moderately to severely active UC who have not been able to respond or poorly responsive to conventional treatment, ADA therapy could be considered. Larger numbers of randomized placebo-controlled trials and multicenter longitudinal studies with longer follow-up periods are needed to determine further the efficacy and safety of ADA.

\section{CONFLICTS OF INTEREST}

No potential conflict of interest relevant to this article was reported.

\section{ACKNOWLEDGEMENTS}

X.L.J. contributed to conception and design of the study. Z.M.Z. and W.L. contributed to conception, design of the study, the data acquisition, statistical analysis, interpretation of the data, and editing the manuscript corporately. All authors commented on drafts of the paper and have approved the final draft of the manuscript.

\section{REFERENCES}

1. Solberg IC, Lygren I, Jahnsen J, et al. Clinical course during the first 10 years of ulcerative colitis: results from a population-based inception cohort (IBSEN Study). Scand J Gastroenterol 2009;44: 431-440.

2. Feuerstein JD, Cheifetz AS. Ulcerative colitis: epidemiology, diag- 
nosis, and management. Mayo Clin Proc 2014;89:1553-1563.

3. Rubin DT, Dubinsky MC, Panaccione R, et al. The impact of ulcerative colitis on patients' lives compared to other chronic diseases: a patient survey. Dig Dis Sci 2010;55:1044-1052.

4. Afif W, Leighton JA, Hanauer SB, et al. Open-label study of adalimumab in patients with ulcerative colitis including those with prior loss of response or intolerance to infliximab. Inflamm Bowel Dis 2009;15:1302-1307.

5. Barreiro-de Acosta M, Lorenzo A, Dominguez-Munoz JE. Adalimumab in ulcerative colitis: two cases of mucosal healing and clinical response at two years. World J Gastroenterol 2009;15:38143816.

6. Peyrin-Biroulet L, Laclotte C, Roblin X, Bigard MA. Adalimumab induction therapy for ulcerative colitis with intolerance or lost response to infliximab: an open-label study. World J Gastroenterol 2007;13:2328-2332.

7. Reinisch W, Sandborn WJ, Hommes DW, et al. Adalimumab for induction of clinical remission in moderately to severely active ulcerative colitis: results of a randomised controlled trial. Gut 2011; 60:780-787.

8. Sandborn WJ, van Assche G, Reinisch W, et al. Adalimumab induces and maintains clinical remission in patients with moderateto-severe ulcerative colitis. Gastroenterology 2012;142:257-265.e3.

9. Suzuki Y, Motoya S, Hanai H, et al. Efficacy and safety of adalimumab in Japanese patients with moderately to severely active ulcerative colitis. J Gastroenterol 2014;49:283-294.

10. U.S. Food and Drug Administration. GIDAC briefing document [Internet]. Silver Spring: U.S. Food and Drug Administration; 2012 [cited 2014 Feb 20]. Available from: http://www.fda.gov/ downloads/advisorycommittees/committeesmeetingmaterials/ drugs/gastrointestinaldrugsadvisorycommittee/ucm323504.pdf.

11. Dignass A, Eliakim R, Magro F, et al. Second European evidencebased consensus on the diagnosis and management of ulcerative colitis. Part 1: definitions and diagnosis. J Crohns Colitis 2012;6:965-990.

12. Guyatt G, Mitchell A, Irvine EJ, et al. A new measure of health status for clinical trials in inflammatory bowel disease. Gastroenterology 1989;96:804-810.

13. Irvine EJ, Feagan B, Rochon J, et al. Quality of life: a valid and reliable measure of therapeutic efficacy in the treatment of inflammatory bowel disease: Canadian Crohn's Relapse Prevention Trial Study Group. Gastroenterology 1994;106:287-296.

14. Tarsilla M. Cochrane handbook for systematic reviews of interventions. J Multidiscip Eval 2010;6:142-148.

15. Jadad AR, Moore RA, Carroll D, et al. Assessing the quality of reports of randomized clinical trials: is blinding necessary? Control Clin Trials 1996;17:1-12.

16. Oussalah A, Laclotte C, Chevaux JB, et al. Long-term outcome of adalimumab therapy for ulcerative colitis with intolerance or lost response to infliximab: a single-centre experience. Aliment Pharmacol Ther 2008;28:966-972.

17. Gies N, Kroeker KI, Wong K, Fedorak RN. Treatment of ulcerative colitis with adalimumab or infliximab: long-term follow-up of a single-centre cohort. Aliment Pharmacol Ther 2010;32:522-528.

18. Feagan BG, Sandborn WJ, Lazar A, et al. Adalimumab therapy is associated with reduced risk of hospitalization in patients with ulcerative colitis. Gastroenterology 2014;146:110-118.e3.

19. Sandborn WJ, Colombel JF, D'Haens G, et al. One-year maintenance outcomes among patients with moderately-to-severely active ulcerative colitis who responded to induction therapy with adalimumab: subgroup analyses from ULTRA 2. Aliment Pharmacol Ther 2013;37:204-213.

20. Vogelaar L, Spijker AV, van der Woude CJ. The impact of biologics on health-related quality of life in patients with inflammatory bowel disease. Clin Exp Gastroenterol 2009;2:101-109.

21. Reinisch W, Sandborn WJ, Panaccione R, et al. 52-Week efficacy of adalimumab in patients with moderately to severely active ulcerative colitis who failed corticosteroids and/or immunosuppressants. Inflamm Bowel Dis 2013;19:1700-1709.

22. Lv R, Qiao W, Wu Z, et al. Tumor necrosis factor alpha blocking agents as treatment for ulcerative colitis intolerant or refractory to conventional medical therapy: a meta-analysis. PLoS One 2014;9:e86692

23. Park SC, Jeen YT. Current and emerging biologics for ulcerative colitis. Gut Liver 2015;9:18-27.

24. Chang CW, Wei SC, Chou JW, et al. Safety and efficacy of adalimumab for patients with moderate to severe Crohn's disease: the Taiwan Society of Inflammatory Bowel Disease (TSIBD) Study. Intest Res 2014;12:287-292.

25. Armuzzi A, Lionetti P, Blandizzi C, et al. Anti-TNF agents as therapeutic choice in immune-mediated inflammatory diseases: focus on adalimumab. Int J Immunopathol Pharmacol 2014;27(1 Suppl): 11-32.

26. Blandizzi C, Gionchetti P, Armuzzi A, et al. The role of tumour necrosis factor in the pathogenesis of immune-mediated diseases. Int J Immunopathol Pharmacol 2014;27(1 Suppl):1-10.

27. Lapadula G, Marchesoni A, Armuzzi A, et al. Adalimumab in the treatment of immune-mediated diseases. Int J Immunopathol Pharmacol 2014;27(1 Suppl):33-48.

28. Nielsen OH, Ainsworth MA. Tumor necrosis factor inhibitors for inflammatory bowel disease. N Engl J Med 2013;369:754-762.

29. Colombel JF, Sandborn WJ, Rutgeerts P, et al. Adalimumab for maintenance of clinical response and remission in patients with Crohn's disease: the CHARM trial. Gastroenterology 2007;132:5265.

30. D'Haens GR, Panaccione R, Higgins PD, et al. The London Position Statement of the World Congress of Gastroenterology on Biological Therapy for IBD with the European Crohn's and Colitis Organization: when to start, when to stop, which drug to choose, and how to predict response? Am J Gastroenterol 2011;106:199-212.

31. Colombel JF, Sandborn WJ, Ghosh S, et al. Four-year maintenance treatment with adalimumab in patients with moderately to severely active ulcerative colitis: data from ULTRA 1, 2, and 3. Am J Gastroenterol 2014;109:1771-1780. 